\title{
Synthetic Fourier transform light scattering
}

\author{
KyeoReh Lee, ${ }^{1}$ Hyeon-Don Kim, ${ }^{2}$ Kyoohyun Kim, ${ }^{1}$ Youngchan Kim, ${ }^{1}$ \\ Timothy R. Hillman, ${ }^{3,4}$ Bumki Min, ${ }^{2}$ and YongKeun Park ${ }^{1, *}$ \\ ${ }^{1}$ Departments of Physics, Korea Advanced Institutes of Science and Technology, Daejeon 305-701, South Korea \\ ${ }^{2}$ Department of Mechanical Engineering, Korea Advanced Institutes of Science and Technology, Daejeon 305-701, \\ South Korea \\ ${ }^{3}$ G. R. Harrison Spectroscopy Laboratory, Massachusetts Institute of Technology, 77 Massachusetts Ave., Cambridge \\ MA 02139, USA \\ ${ }^{4}$ Current mailing address: PO Box 83, Parkville, Victoria, 3052 Australia \\ *yk.park@kaist.ac.kr
}

\begin{abstract}
We present synthetic Fourier transform light scattering, a method for measuring extended angle-resolved light scattering (ARLS) from individual microscopic samples. By measuring the light fields scattered from the sample plane and numerically synthesizing them in Fourier space, the angle range of the ARLS patterns is extended up to twice the numerical aperture of the imaging system with unprecedented sensitivity and precision. Extended ARLS patterns of individual microscopic polystyrene beads, healthy human red blood cells (RBCs), and Plasmodium falciparumparasitized RBCs are presented.
\end{abstract}

C2013 Optical Society of America

OCIS codes: (090.2880) Holographic interferometry; (170.1530) Medical optics and biotechnology: Cell analysis; (290.5820) Scattering measurements.

\section{References and links}

1. H. C. d. Hulst, Light scattering by small particles (Wiley, New York, 1957).

2. A. A. Kokhanovsky, Optics of light scattering media: problems and solutions (John Wiley: Praxis, Chichester; New York, 1999).

3. H. Ding, Z. Wang, F. Nguyen, S. A. Boppart, and G. Popescu, "Fourier transform light scattering of inhomogeneous and dynamic structures," Phys. Rev. Lett. 101(23), 238102 (2008).

4. H. Ding, F. Nguyen, S. A. Boppart, and G. Popescu, "Optical properties of tissues quantified by Fouriertransform light scattering," Opt. Lett. 34(9), 1372-1374 (2009).

5. H. Ding, Z. Wang, F. T. Nguyen, S. A. Boppart, L. J. Millet, M. U. Gillette, J. Liu, M. D. Boppart, and G. Popescu, "Fourier Transform Light Scattering (FTLS) of Cells and Tissues," J. Com. Theo. Nanosci. 7(12), 2501-2511 (2010).

6. Y. Park, C. A. Best-Popescu, R. R. Dasari, and G. Popescu, "Light scattering of human red blood cells during metabolic remodeling of the membrane," J. Biomed. Opt. 16(1), 011013 (2011).

7. Y. Park, M. Diez-Silva, D. Fu, G. Popescu, W. Choi, I. Barman, S. Suresh, and M. S. Feld, "Static and dynamic light scattering of healthy and malaria-parasite invaded red blood cells," J. Biomed. Opt. 15(2), 020506 (2010).

8. Y. Kim, J. M. Higgins, R. R. Dasari, S. Suresh, and Y. K. Park, "Anisotropic light scattering of individual sickle red blood cells," J. Biomed. Opt. 17(4), 040501 (2012).

9. H. Ding, L. J. Millet, M. U. Gillette, and G. Popescu, "Actin-driven cell dynamics probed by Fourier transform light scattering," Biomed. Opt. Express 1(1), 260-267 (2010).

10. H. Yu, H. Park, Y. Kim, M. W. Kim, and Y. Park, "Fourier-transform light scattering of individual colloidal clusters," Opt. Lett. 37(13), 2577-2579 (2012)

11. C. Cho, H. Kim, S. Jeong, S.-W. Baek, J.-W. Seo, D. Han, K. Kim, Y. Park, S. Yoo, and J.-Y. Lee, "Random and V-groove texturing for efficient light trapping in organic photovoltaic cells," Sol. Energy Mater. Sol. Cells 115, 36-41 (2013).

12. W. Choi, C. C. Yu, C. Fang-Yen, K. Badizadegan, R. R. Dasari, and M. S. Feld, "Field-based angle-resolved light-scattering study of single live cells," Opt. Lett. 33(14), 1596-1598 (2008).

13. K. Kim and Y. Park, "Fourier transform light scattering angular spectroscopy using digital inline holography," Opt. Lett. 37(19), 4161-4163 (2012).

14. W. M. Brown, "Synthetic aperture radar," IEEE Trans. Aerospace Electron. Syst. 2(2), 217-229 (1967).

15. M. Neil and A. Squire, "Wide-field optically sectioning fluorescence microscopy with laser illumination," J. Microsc. 197(1), 1-4 (2000).

16. S. A. Alexandrov, T. R. Hillman, T. Gutzler, and D. D. Sampson, "Synthetic aperture Fourier holographic optical microscopy," Phys. Rev. Lett. 97(16), 168102 (2006).

17. L. Martínez-León and B. Javidi, "Synthetic aperture single-exposure on-axis digital holography," Opt. Express 16(1), 161-169 (2008). 
18. M. Paturzo, F. Merola, S. Grilli, S. De Nicola, A. Finizio, and P. Ferraro, "Super-resolution in digital holography by a two-dimensional dynamic phase grating," Opt. Express 16(21), 17107-17118 (2008).

19. V. Mico, Z. Zalevsky, P. García-Martínez, and J. García, "Synthetic aperture superresolution with multiple offaxis holograms," J. Opt. Soc. Am. A 23(12), 3162-3170 (2006).

20. V. Mico, Z. Zalevsky, and J. Garcia, "Synthetic aperture microscopy using off-axis illumination and polarization coding," Opt. Commun. 276(2), 209-217 (2007).

21. V. Micó, Z. Zalevsky, C. Ferreira, and J. García, "Superresolution digital holographic microscopy for threedimensional samples," Opt. Express 16(23), 19260-19270 (2008).

22. T. R. Hillman, T. Gutzler, S. A. Alexandrov, and D. D. Sampson, "High-resolution, wide-field object reconstruction with synthetic aperture Fourier holographic optical microscopy," Opt. Express 17(10), 7873-7892 (2009).

23. M. Born and E. Wolf, Principles of Optics: Electromagnetic Theory of Propagation, Interference and Diffraction of Light (Cambridge University, 1999).

24. T. M. Habashy, R. W. Groom, and B. R. Spies, "Beyond the Born and Rytov approximations: A nonlinear approach to electromagnetic scattering," J. Geophys. Res.: Solid Earth (1978-2012) 98(B2), 1759-1775 (1993).

25. K. Kim, H. Yoon, M. Diez-Silva, M. Dao, R. R. Dasari, and Y. Park, "High-resolution three-dimensional imaging of red blood cells parasitized by Plasmodium falciparum and in situ hemozoin crystals using optical diffraction tomography," J. Biomed. Opt. 19(1), 011005 (2014).

26. Y. J. Sung, W. Choi, C. Fang-Yen, K. Badizadegan, R. R. Dasari, and M. S. Feld, "Optical diffraction tomography for high resolution live cell imaging," Opt. Express 17(1), 266-277 (2009).

27. V. Lauer, "New approach to optical diffraction tomography yielding a vector equation of diffraction tomography and a novel tomographic microscope," J. Microsc. 205(2), 165-176 (2002).

28. A. J. Devaney, "Inverse-scattering theory within the Rytov approximation," Opt. Lett. 6(8), 374-376 (1981).

29. K. Lee, K. Kim, J. Jung, J. H. Heo, S. Cho, S. Lee, G. Chang, Y. J. Jo, H. Park, and Y. K. Park, "Quantitative phase imaging techniques for the study of cell pathophysiology: from principles to applications," Sensors (Basel) 13(4), 4170-4191 (2013).

30. M. Madou, Fundamentals of microfabrication: the science of miniaturization (CRC press, 2002).

31. Y. Park, M. Diez-Silva, G. Popescu, G. Lykotrafitis, W. Choi, M. S. Feld, and S. Suresh, "Refractive index maps and membrane dynamics of human red blood cells parasitized by Plasmodium falciparum," Proc. Natl. Acad. Sci. U.S.A. 105(37), 13730-13735 (2008).

32. G. Pasvol, R. J. Wilson, M. E. Smalley, and J. Brown, "Separation of viable schizont-infected red cells of Plasmodium falciparum from human blood," Ann. Trop. Med. Parasitol. 72(1), 87-88 (1978).

33. C. Lambros and J. P. Vanderberg, "Synchronization of Plasmodium falciparum erythrocytic Stages in Culture," J. Parasitol. 65(3), 418-420 (1979).

34. T. Rothe, M. Schmitz, and A. Kienle, "Angular and spectrally resolved investigation of single particles by darkfield scattering microscopy," J. Biomed. Opt. 17(11), 117006 (2012).

35. M. Diez-Silva, Y. Park, S. Huang, O. Mercereau-Puijalon, G. Deplaine, C. Lavazec, S. Perrot, S. Bonnefoy, M. Dao, M. S. Feld, J. Han, and S. Suresh, "Pf155/RESA protein influences the dynamic microcirculatory behavior of ring-stage Plasmodium falciparum infected red blood cells," Sci. Rep. (2012).

36. H. S. Byun, T. R. Hillman, J. M. Higgins, M. Diez-Silva, Z. Peng, M. Dao, R. R. Dasari, S. Suresh, and Y. K. Park, "Optical measurement of biomechanical properties of individual erythrocytes from a sickle cell patient," Acta Biomat. (2012).

37. R. Chandramohanadas, Y. Park, L. Lui, A. Li, D. Quinn, K. Liew, M. Diez-Silva, Y. Sung, M. Dao, C. T. Lim, P. R. Preiser, and S. Suresh, "Biophysics of malarial parasite exit from infected erythrocytes," PLoS ONE 6(6), e20869 (2011).

\section{Introduction}

Angle resolved light scattering (ARLS) provides information about the morphological and optical properties of scatterers, and it has been widely used in the fields of biology, chemistry, meteorology, and astronomy [1,2]. Fourier transform light scattering (FTLS) is a recently developed technique that enables the effective measurement of the ARLS signal from individual microscopic samples with unprecedented sensitivity [3]. In FTLS, the scattered light field from a sample plane is holographically measured and then numerically propagated to the far field, that is, the Fourier plane, to calculate the ARLS pattern from the sample. The capability and effectiveness of the FTLS has been shown in various applications in diverse fields: biological tissues [4,5]; cells [6-9]; colloidal clusters [10]; and light trapping material for solar cells [11]. However, the range of scattered angles that can be measured in FTLS is limited by the numerical aperture (NA) of optical imaging systems; light from the sample scattered at large angles is inaccessible. This limited range is unfortunate because FTLS has much to offer the field of microscopy and spectroscopy with its single-shot measurement nature, extremely high sensitivity, and capability of targeting individual microscopic samples. Furthermore, the high-angle-scattered light usually contains information from submicroscopic sample structures. 
Here we present a novel method, synthetic Fourier transform light scattering (sFTLS), to overcome the limited angle issue in FTLS. We demonstrate, by holographically synthesizing multiple scattered fields obtained with different angles of illumination, the two-dimensional ARLS signal be extended up to twice the NA of imaging system. In addition, the signal-tonoise ratio (SNR) of the sFTLS signal is dramatically increased due to the acquisition of multiple exposures between which the noise is uncorrelated. The measurements of large-angle light scattering patterns are demonstrated using individual polystyrene beads and SU-8 photoresist disks. We also present light scattering measurements from individual live human red blood cells (RBCs) and Plasmodium falciparum-parasitized RBCs (Pf-RBCs).

\section{Theory and simulations}

\subsection{Fourier transform light scattering (FTLS)}

The principle of FTLS is to first measure the scalar electric field (E-field) at the image plane and then numerically propagate it to the far field using the Fourier transform operation $[3,12]$. The E-field at the image plane can be measured using quantitative phase microscopy. Recently, it has been shown that conventional microscopy equipped with coherent illumination can also be used for FTLS [13].

The propagation of the scattered field from a sample in the $z$-direction can be described by the paraxial approximation to Helmholtz's equation:

$$
\nabla^{2} U(\mathbf{r})+2 i k \frac{\partial}{\partial z} U(\mathbf{r})=0
$$

where $U(\mathbf{r})$ is the complex amplitude of the scattered E-field at a transverse position $\mathbf{r}=$ $(x, y), k$ is the wavenumber, and $\nabla^{2}$ is the transverse part of the Laplacian operator. The farfield solution of Eq. (1) is well known as Fraunhofer diffraction,

$$
U_{z}(x, y)=\frac{1}{i \lambda z} \exp \left[-i k\left(\frac{x^{2}+y^{2}}{2 z}\right)\right] \iint U_{0}\left(x_{0}, y_{0}\right) \exp \left[-i k \frac{\left(x x_{0}+y y_{0}\right)}{z}\right] d x_{0} d y_{0},
$$

where $U_{z}(x, y)$ is the far-field propagation of the initial field $U_{0}\left(x_{0}, y_{0}\right)$ and $\lambda$ is the wavelength. This field distribution $U_{z}(x, y)$ is directly proportional to the 2D Fourier transformation of an initial E-field, $\tilde{U}_{0}(\xi, \eta)=F T\left[U_{0}\left(x_{0}, y_{0}\right)\right]$, where

$$
\xi=\frac{2 \pi n_{m}}{\lambda} \sin \theta \cos \phi, \eta=\frac{2 \pi n_{m}}{\lambda} \sin \theta \sin \phi
$$

represent spatial frequencies along $x$ - and $y$-directions, respectively. The refractive index (RI) of the medium is denoted $n_{m}$, and $\theta, \phi$ are polar and azimuthal angles of the scattered vector, respectively. Even though Eq. (2) is, strictly speaking, only valid in the paraxial approximation, there is, more generally, a one-to-one correspondence between transverse spatial frequency and scattering angle. Thus, the simple Fourier transformation of the E-field $U_{0}\left(x_{0}, y_{0}\right)$ measured at the image plane gives the ARLS spectrum of the sample (up to an angle-dependent correction factor).

\subsection{Synthetic Fourier transform light scattering (sFTLS)}

Here we present the concept of sFTLS. sFTLS measures extended ARLS by numerically synthesizing the scattered light signals acquired with different angles of illumination. In sFTLS, the angle range of ARLS can be extended up to twice the NA of imaging system. The

\#194456 - \$15.00 USD Received 23 Jul 2013; revised 6 Sep 2013; accepted 7 Sep 2013; published 16 Sep 2013 (C) 2013 OSA 23 September 2013 | Vol. 21, No. 19 | DOI:10.1364/OE.21.022453 | OPTICS EXPRESS 22455 
principle of sFTLS is illustrated in Fig. 1. In FTLS, the maximum angle of the scattered vector $\theta_{\max }$ is fundamentally determined by the NA of the optical imaging system:

$$
N A=n_{m} \sin \theta_{\max },
$$

where $n_{m}$ is the refraction index of the medium in which the lens is situated. As shown in Fig. 1(A), a scatterer is illuminated with a coherent plane wave, and only a fraction of the scattered field, limited by the NA of an objective lens, is obtained. Therefore, the range of the FTLS signal in Fourier space is limited by a circle with a radius of $\left|k_{N A}\right|=2 \pi \mathrm{NA} / \lambda$.

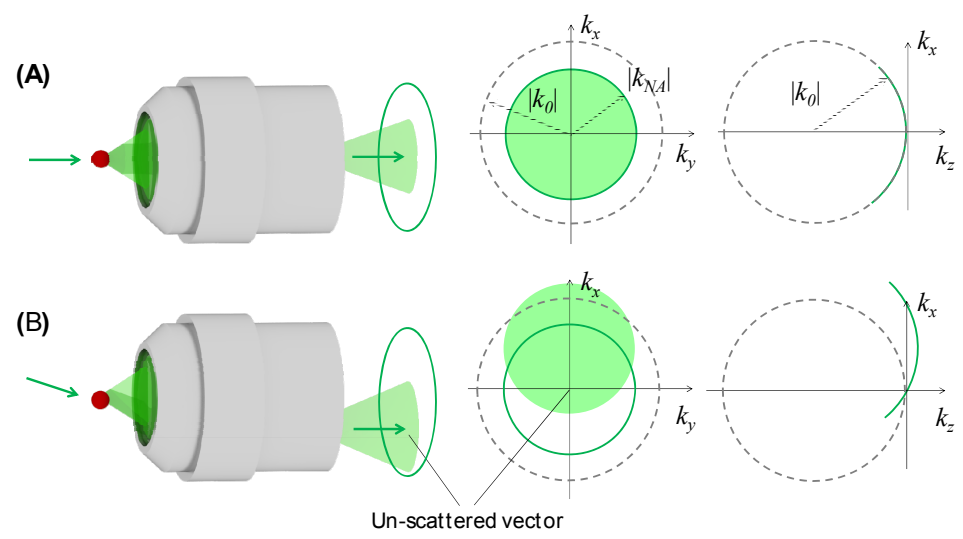

(C)
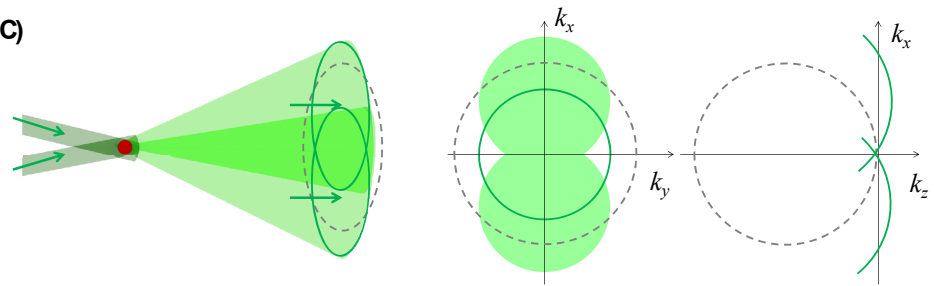

Fig. 1. Illustrations of light scattered field at image and Fourier space with: (A) a normal angle of illumination; (B) an oblique angle of illumination; and (C) synthesized aperture with two different illuminations. Acceptable scattered light is limited by the NA of an objective lens, depicted as filled circles. Dotted rings depict the cross-sections of Ewald spheres in the normal illumination case [23].

To overcome this angle limitation, sFTLS employs the principle of the aperture synthesis. This principle was first introduced in the field of synthetic aperture radar [14], and then widely utilized in microscopic imaging techniques in order to increase spatial resolving power (for example, structured illumination microscopy [15], synthetic aperture holographic optical microscopy [16-18], and super-resolution digital holographic microscopy [19]).

The scattered E-fields obtained with different angles of illumination permit the highspatial-frequency information beyond the NA of the imaging system to be delivered, which is captured holographically and synthesized in Fourier space. This corresponds to the expansion of the angle-range of the ARLS signal, beyond that which is ordinarily accessible. As shown in Fig. 1(B), the scattered E-field obtained with a specific off-axis illumination angle is still limited by the NA. Nonetheless, due to the shifted input spatial frequency $\left(\xi_{0}, \eta_{0}\right)$ due to the specific off-axis illumination, the region of the Fourier spectrum corresponding to this E-field is not identical to that due to a normal illumination angle. As a result, it is possible to access high-spatial-frequency components beyond the NA of the system. 
In sFTLS, multiple E-fields $U_{0}\left(x_{0}, y_{0} ; \xi_{0}, \eta_{0}\right)$ scattered from a sample are first measured with different angles of illumination $\left(\xi_{0}, \eta_{0}\right)$. Then, the corresponding Fourier spectra of the E-fields $\tilde{U}_{0}\left(\xi, \eta ; \xi_{0}, \eta_{0}\right)$ are laterally translated to ensure that the spectra are in the same coordinate system, so that they are represented $\tilde{U}_{0}\left(k_{x}, k_{y} ; \xi_{0}, \eta_{0}\right)$, where the indices are defined as

$$
k_{x}=\xi-\xi_{0}, k_{y}=\eta-\eta_{0}
$$

Then these scattering spectra are stitched and synthesized into an angular scattering distribution with an extended angle of acceptance:

$$
\hat{U}\left(k_{x}, k_{y}\right)=\sum_{\left(\xi_{0}, \eta_{0}\right)} \tilde{U}_{0}\left(k_{x}, k_{y} ; \xi_{0}, \eta_{0}\right) .
$$

\subsection{Selective synthesis}

Given the illumination wave direction, each component of the scattered wave Fourier spectrum corresponds to a particular 3D sample spatial frequency. Its $z$-component is:

$$
k_{z}=\sqrt{k_{0}^{2}-\xi^{2}-\eta^{2}}-\sqrt{k_{0}^{2}-\xi_{0}^{2}-\eta_{0}^{2}},
$$

where $k_{0}=2 \pi n_{m} / \lambda$ represents the radius of Ewald's sphere, given the RI of a medium $n_{m}$. As is implicit from Eq. (7), two points in spatial frequency space sharing the same $\left(k_{x}, k_{y}\right)$ pair, but accessible with different illumination angles, will have different $k_{z}$ components. This becomes problematic for aperture synthesis with a sample of finite thickness, especially for large scattering angles.

The light fields scattered from a thick sample cannot be precisely synthesized by simply stitching at Fourier space as is done in conventional synthetic aperture approaches. This issue has been implied by previous research in which samples were required to be thin [19-21], and explicitly pointed out by Hillman et al. [22], which utilized a reflection geometry, the $k_{z}$ displacement between the spatial frequency regions acquired with different polar illumination angles necessitated that polar angle be fixed, and only azimuthal angle varied to generate an annular synthetic aperture. For the wide-field imaging application, this provided an advantage. However, for our purpose, such an approach would unnecessarily trade off the low-frequency information of the sample. We use a transmission geometry, for which the $k_{z^{-}}$ variation between different acquisitions is much less severe: indeed, all interferograms contain some information in 3D Fourier space within the vicinity of the region $k_{z}=0$. We describe how to exploit this fact in the following paragraphs.

To systematically address this issue of aperture synthesis in sFTLS, we define thin-sample limit $\sigma$ of a sample with a crude maximum thickness of $h$, as follows:

$$
\sigma=\frac{\pi}{k_{0} h}
$$

The dimensionless parameter $\sigma$ serves as a criterion of thinness to guarantee the validity of the aperture synthesis. Intuitively, $\sigma$ can be understood as the optical thickness of a sample that is normalized by wavelength of the light.

Within a certain range determined with $\sigma$, measured scattered far-fields can be approximated as $\hat{F}\left(k_{x}, k_{y}, 0\right)$, that is, $\hat{F}\left(k_{x}, k_{y},\left|k_{z}\right|<\sigma k_{0}\right) \cong \hat{F}\left(k_{x}, k_{y}, 0\right)$. Alternatively expressed, within the range $\left|k_{z}\right|<\sigma k_{0}, \hat{F}\left(k_{x}, k_{y}, k_{z}\right)$ is strongly correlated with $\hat{F}\left(k_{x}, k_{y}, 0\right)$. In other words, using the limited fraction of each scattering spectrum satisfying $\left|k_{z}\right|<\sigma k_{0}$,

\#194456 - \$15.00 USD Received 23 Jul 2013; revised 6 Sep 2013; accepted 7 Sep 2013; published 16 Sep 2013 (C) 2013 OSA 23 September 2013 | Vol. 21, No. 19 | DOI:10.1364/OE.21.022453 | OPTICS EXPRESS 22457 
sFTLS signals can be effectively and precisely synthesized from scattered E-fields with different angles of illumination, a process we call selective synthesis.

To demonstrate the validity of sFTLS with selective synthesis, we performed numerical simulations (Fig. 2) using three phantoms with uniform optical properties: a thin disk; a thick disk; and a thick sphere. Employing the First Rytov approximation [23], we preset the 3D RI distribution of the sample $n_{s}(\mathbf{r})$, and calculated the corresponding 3-D scattering potentials $F(\mathbf{r})=\frac{k_{0}^{2}}{4 \pi}\left[\left(n_{s}(\mathbf{r}) / n_{m}\right)^{2}-1\right]$. From $F(\mathbf{r})$, we derived expected results for the far-field scattering patterns of each sample. These simulations represent the inverse operation of constructing 3D RI tomogram from angle-varying holographic results. Since this simulation is based upon the First Rytov approximation, it cannot predict the real result precisely. However, in cases where $F(\mathbf{r})$ varies slowly over space such as for uniform beads, uniform disks and almost all biological samples, it has been shown that the First Rytov approximation represents quite reliable results, compared with exact solutions [24].

(A)

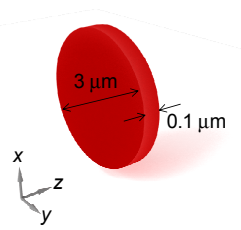

(B)

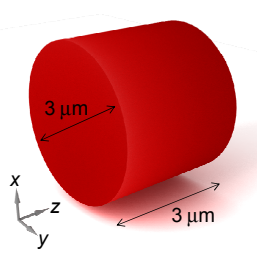

(C)

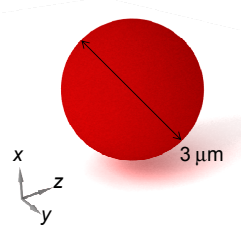

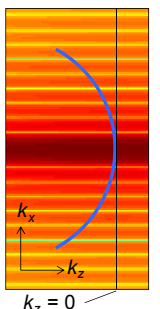
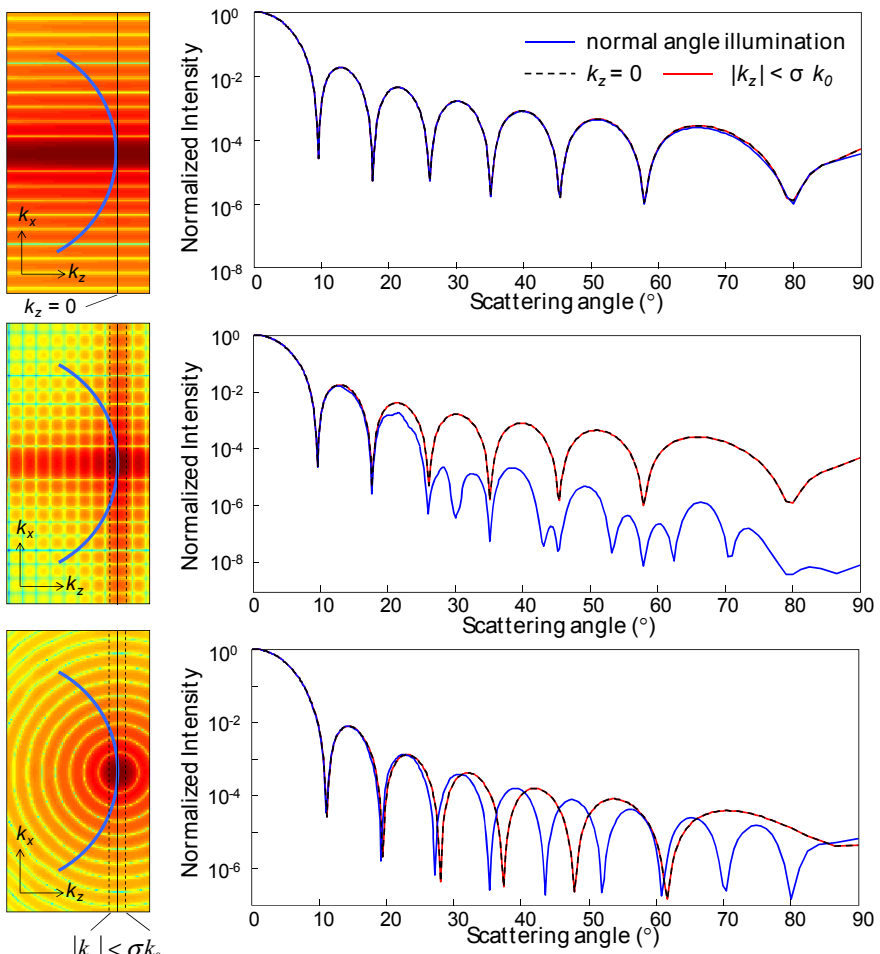

Fig. 2. Simulated light scattering potentials and synthesized ARLS patterns of: (A) a thin disk; (B) a thick disk; and (C) a think sphere. The parameters $n_{s}=1.587, n_{m}=1.511, \lambda=633 \mathrm{~nm}$ for all cases. Cross-sections of the scattered potential at $k_{y}=0$ are depicted in the middle column; the solid blue arcs depict the fraction of Ewald's surfaces for the normal illumination with NA $=1.4$. The right column shows the sFTLS signals for various conditions. Blue lines: Angular spectra for normal illumination. Red lines: spectrum within the thin-sample limit. Dotted black lines: scattering spectra within the plane $k_{z}=0$.

The Rytov approximation was used in this study, since it works independently of the sample size, whereas the Born approximation depends on the sample size; the samples used in this study are optically transparent, but are thicker than wavelength of light. In some cases such as for thinner samples with the same order of optical complexity, the First Born approximation shows more reliable results; the choice of approximation in the reconstruction should be carefully considered $[25,26]$. 
For a thin sample [Fig. 2(A)], the sFTLS signals obtained with and without the selective aperture synthesis do not show significant difference from each other, and both sFTLS signals are comparable with $\hat{F}\left(k_{x}, k_{y}, 0\right)$. Ideally, for a sample with zero thickness, i.e., for which $F(\mathbf{r})=F(x, y) \delta(z), \hat{F}\left(k_{x}, k_{y}, k_{z}\right)$ has a constant value along $k_{z}$-axis. Therefore, all points with the same $\left(k_{x}, k_{y}\right)$ pair carry identical information. However, the sFTLS signals from thick samples without the selective aperture synthesis show significant deviation from $\hat{F}\left(k_{x}, k_{y}, 0\right)$, and this deviation becomes greater for large scattering angles [Figs. 2(B) and (C)]. For thick samples, the sFTLS signals with selective aperture synthesis show good agreement with the expected results (scattering spectra within the plane $k_{z}=0$ ).

In addition, the validity of the criterion of thinness can be checked with the observation that the first minimas of the $z$-directional autocorrelations for $\hat{F}\left(k_{x}, k_{y}, k_{z}\right)$ for the thick samples in Figs. 2(B) and 2(C), are equal to $2 \pi / k_{0} h$ and $1.43\left(2 \pi / k_{0} h\right)$, respectively.

In the Fig. 2, we display all graphs in the form of angle versus intensity to compare with the FTLS results. However, using sFTLS with selective synthesis, we can even measure the information beyond $90^{\circ}$ along the scanning axis, $\left|k_{x}\right|>k_{0}$, which cannot be represented by an angle-intensity plot due to the finite domain of arcsine [See also Fig. 1(C)].

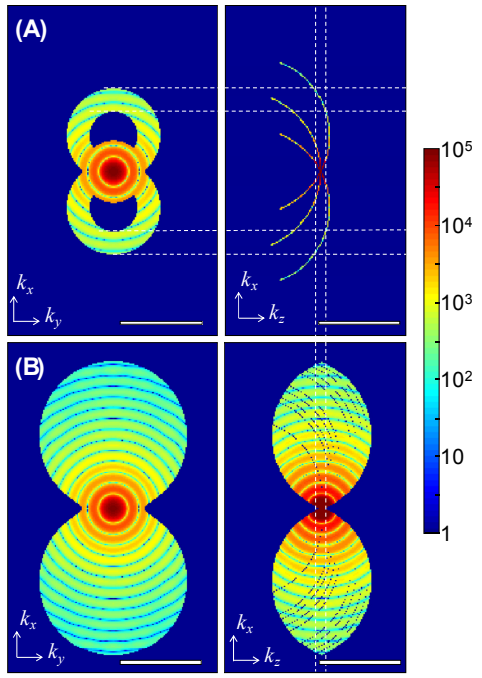

(C)

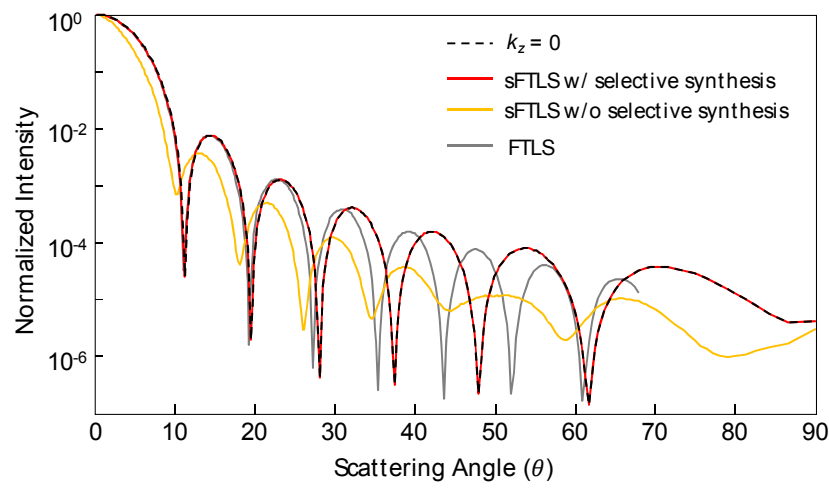

Fig. 3. Simulated sFTLS with the selective synthesis. The sFTLS signals from a phantom sphere are synthesized with (A) 3 illumination angles and (B) 600 illuminations angles. Vertical dotted lines represent a range for the selective synthesizing, and corresponding width after synthesis (horizontal). Scale bars indicate $2.5 \mu \mathrm{m}^{-1}$ (C) Various simulation results of the same phantom sphere. Only sFTLS with selective synthesis matches with $k_{z}=0$ information.

The detailed procedure of sFTLS is shown in Fig. 3. Figure 3(A) shows the ARLS patterns synthesized with three representative FTLS spectra obtained with different angles of illumination. Only the fractions of each spectrum satisfying the condition $\left|k_{z}\right|<\sigma k_{0}$ are utilized for the selective aperture synthesis. Figure 3(B) shows the final sFTLS pattern after synthesizing 600 FTLS signals. For simplicity and computational efficiency, we set the $y$-axis as a pair of rotation coordinates $(\phi=0, \pi)$. We also simulated FTLS and sFTLS without selective synthesis, and sFTLS with selective synthesis for same phantom sphere (Fig. 3(C)). The results clearly show a fundamental limit of FTLS and problem of the conventional synthesizing method. 
In summary, we have shown that the conventional holographic synthesis method has systematic problems for thick samples, since it accesses regions of a $3 \mathrm{D}$, not a $2 \mathrm{D}$ Fourier map, a fact that has been used for 3D RI tomogram reconstruction, considering diffraction [27]. To manage the issue, we have proposed a selective synthesis method, defining the "thin region' $\left(\left|k_{z}\right|<\sigma k_{0}\right)$ of the 3D Fourier map. It is noteworthy that this thin region is identical to the 2D Fourier transform of the projection of the sample onto a single $x y$ image plane [28].

\section{Experimental results}

\subsection{Instrumentation}

To measure FTLS signals with different angle of illumination, we have used a quantitative phase imaging system equipped with a two-axis galvano-mirror device (See Ref [29]. for the details). Either a diode-pumped solid state (DPSS) laser $(\lambda=532 \mathrm{~nm}, 100 \mathrm{~mW}$, SDL-532$100 \mathrm{~T}$, Shanghai Dream Laser, China) or a He-Ne laser $(\lambda=633 \mathrm{~nm}, 5 \mathrm{~mW}$, HNL050R, Thorlabs, US) was first spatially filtered and used as an illumination source. The two-axis galvano-mirror device (GVS012/M, Thorlabs, US) was located at a plane conjugate to the back-focal-plane of a condenser lenses $(60 \times, \mathrm{NA}=0.9$, UPLFL N, Olympus, Japan) and controlled the illumination angle of the beam by changing the angle of the $x$ - and $y$-axes of the mirrors. The high NA objective lens $(100 \times, \mathrm{NA}=1.4$, oil-immersion, UPLSAPO 100XO, Olympus, Japan) collected the diffracted beam from a sample. The diffracted beam from individual samples was then projected to the image plane where it interfered with a reference wave with slight off-axis illumination to form a spatially modulated interferogram. A CMOS (complementary metal oxide) camera (FASTCAM 1024 PCI, Photron, Japan) was used to record the interferograms. By varying the angle of illumination from $\theta=+35^{\circ}$ to $\theta=-35^{\circ}$ (inside of medium, $n_{m}=1.511, \lambda=532 \mathrm{~nm}$ ) by the galvanometer.

To construct one aperture-synthesized scattering map, we typically recorded 600 interferograms at the acquisition rate of $125 \sim 5,000$ frames/sec. Processing time for the reconstruction was approximately 1 min for 600 interferograms $(384 \times 384$ pixels), using a 64-bit $\mathrm{MatLab}^{\mathrm{TM}}$ with a personal computer (Intel Core i5-3550 3.3GHz, 8 GB RAM memory.)

\section{2 sFTLS of microscopic transparent objects}

To demonstrate the capability of sFTLS, we first measured individual microscopic transparent objects: a polystyrene bead with diameter of $3.6 \mu \mathrm{m}$ and $n_{s}=1.5986$ (79166, Sigma-Aldrich Inc.); and a photoresist (PR) disk with diameter of $3 \mu \mathrm{m}$, thickness of $1 \mu \mathrm{m}$, and $n_{s}=1.599$. These samples were chosen as test objects because they are physically robust and have sizes and optical properties comparable with the phantoms used in the theory and simulation discussed in Section 2.

The PR disks of SU-8 (an epoxy-based negative photoresist) are fabricated by a conventional MEMS technology [30]. In order to peel off PR disks from a silicon substrate, a $300 \mathrm{~nm}$ thick silver layer is used as a sacrificial layer. After development, the silicon wafer is immersed in SC-1 $\left(1 \mathrm{H}_{2} \mathrm{O}_{2}: 1 \mathrm{NH}_{4} \mathrm{OH}: 5\right.$ de-ionized water in volume ratio) for 3 minutes to etch the silver layer. Individual micro-structures immersed into oil $\left(n_{m}=1.519\right.$ at $532 \mathrm{~nm}$, IMMOIL-F30CC, Olympus) in order to reduce diffraction are sandwiched via two cover glasses.

The diffracted beams were measured with the off-axis interferometer using the DPSS laser $(\lambda=532 \mathrm{~nm})$ described in Section 3.1. For the same sample, we recorded 600 interferograms corresponding to the different angles of illumination. The conventional FTLS signal is obtained from normal angle of illumination and the sFTLS signal is synthesized from the 600 interferograms as described in Section 2.3.

\#194456 - \$15.00 USD Received 23 Jul 2013; revised 6 Sep 2013; accepted 7 Sep 2013; published 16 Sep 2013 (C) 2013 OSA 23 September 2013 | Vol. 21, No. 19 | DOI:10.1364/OE.21.022453 | OPTICS EXPRESS 22460 
(A)

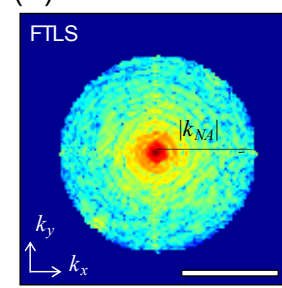

(B)
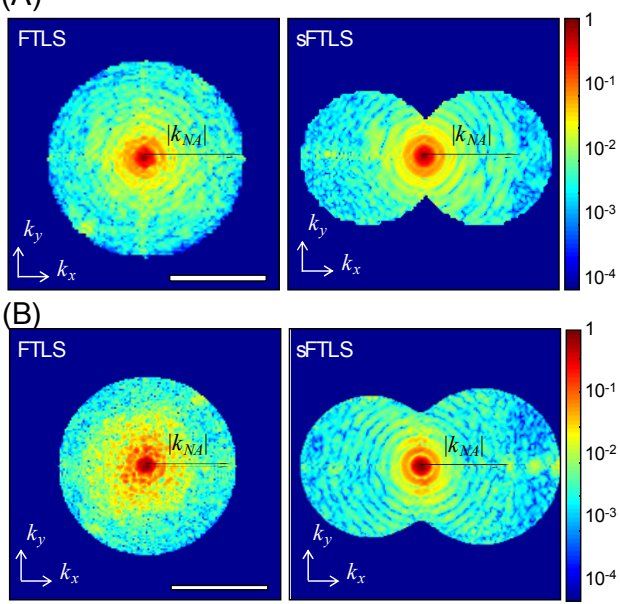

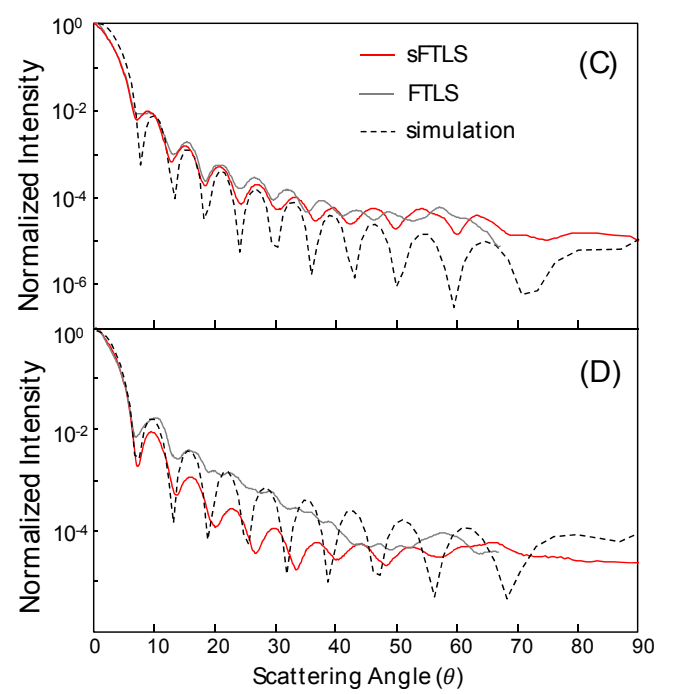

Fig. 4. Experimental results. ARLS signals of (A) a polystyrene bead (diameter $=3.6 \mu \mathrm{m}$ ) with $n_{s}=1.5986$ and (B) a SU-8 photoresist disk (diameter $\times$ thickness $=3 \mu \mathrm{m} \times 1 \mu \mathrm{m}$ ) with $n_{s}=$ 1.599 immersed in oil, $n_{m}=1.519, \lambda=532 \mathrm{~nm}$. The first and second columns show the light scattering spectra in Fourier space obtained with FTLS and sFTLS, respectively. Scale bars indicate $2.5 \mu^{-1}$. (C) and (D) The light scattering spectra along the scattering angle for the polystyrene bead in (A) and the photoresist disk in (B), respectively. The simulation graphs are calculated in the manner of Fig. 3.

The FTLS and sFTLS signals for individual beads and disks are shown in Fig. 4(A) and 4(B), respectively. In FTLS, the maximum observable angle is fundamentally limited by the NA of the objective lens, which is $68^{\circ}$ in the present experiment. However, in sFTLS, the maximum observable angle is $90^{\circ}$ (and even beyond theoretically), since sFTLS systematically synthesize the NA and expand the maximum observable angle.

Also noteworthy is the fact that the signal-to-noise ratio (SNR) in the sFTLS signals is significantly enhanced in comparison with the one in the FTLS. For example, the sFTLS of a polystyrene bead exhibits characteristic oscillatory features in the scattering spectrum up to $65^{\circ}$, whereas the FTLS signal shows an oscillatory spectrum only up to $55^{\circ}$. For a weak scattering sample such as the individual SU-8 microdisks, the effect of enhanced SNR becomes more significant. The sFTLS of an SU-8 microdisk shows the oscillatory features in the scattering spectrum up to $65^{\circ}$ ( 9 peaks), whereas the FTLS signal shows oscillatory spectrum only up to $20^{\circ}$ (3-4 peaks.) The increased SNR in sFTLS results from the synthesis of multiple apertures; a set of replicate measurements are averaged for scattering spectra. In principle, the SNR will be increased in proportion to the square root of the number of measurements. Therefore, the sFTLS signal, synthesized from $N$ scattering spectra obtained with different angles of illumination, would have an SNR increased by a factor of $\sqrt{N}$ at $0^{\circ}$, that will reduce to unity at the maximum angle of acceptance.

\section{3 sFTLS of biological samples}

In order to demonstrate the capability of sFTLS, we used it to measure individual biological samples. The sFTLS signals of a RBC and a $P f$-RBC were measured. RBCs and $P f$-RBCs were prepared following the standard protocol [31]. RBCs from a healthy donor were collected in vacutainer tubes containing an anticoagulant (ethylenediaminetetraacetic acid or EDTA), which were centrifuged at $2000 \mathrm{~g}$ for $10 \mathrm{~min}$ at $10^{\circ} \mathrm{C}$ to separate the $\mathrm{RBCs}$ from the surrounding plasma. The RBCs were then washed three times with phosphate-buffered saline (PBS). After washing, they were diluted in PBS to approximately $10^{6} \mathrm{RBC}$ per $\mathrm{ml}$ prior to the experiments. P. falciparum 3D7A were maintained in leukocyte-free human $\mathrm{O}+$ erythrocytes 
(Research Blood Components) under an atmosphere of $3 \% \mathrm{O}_{2}, 5 \% \mathrm{CO}_{2}$, and $92 \% \mathrm{~N}_{2}$ in RPMI medium 1640 (Gibco Life Technologies) supplemented with $25 \mathrm{mM}$ Hepes (Sigma), $200 \mathrm{mM}$ hypoxanthine (Sigma), 0.209\% NaHCO3 (Sigma), and 0.25\% albumax I (Gibco Life Technologies). Cultures were synchronized successively by concentration of mature schizonts by using plasmagel flotation [32] and sorbitol lysis 2 hours after merozoite invasion to remove residual schizonts [33].

(A)

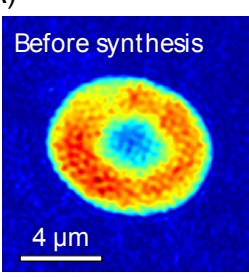

(B)

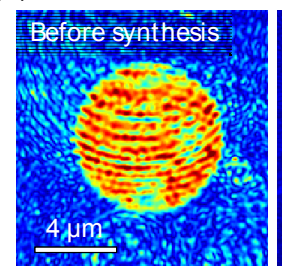

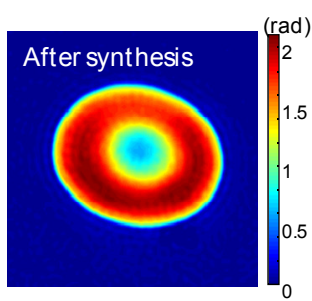

(C)

$$
\text { (C) }
$$

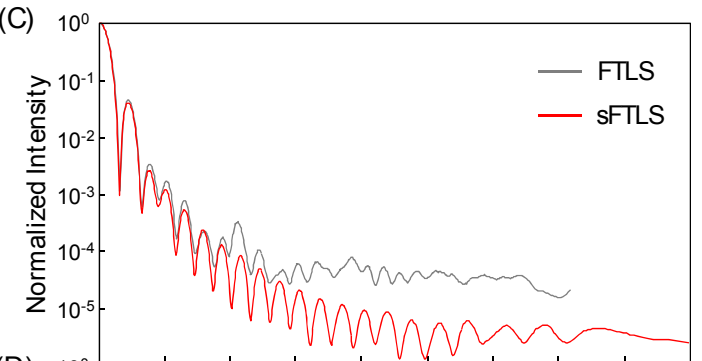

(D)

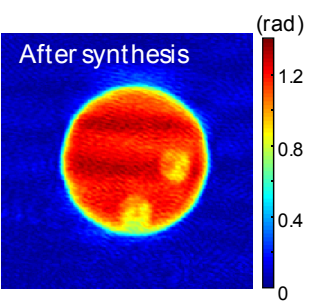

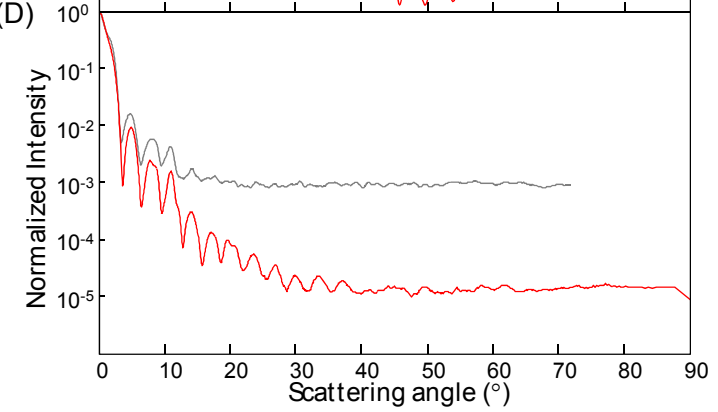

Fig. 5. Experimental results. ARLS signals of a healthy and a $P f$-RBC at ring stage. (A) and (B) show the quantitative phase images of a healthy and a $P f$-RBC at ring stage, respectively. The first column shows the before-and-after-synthesis quantitative phase imaging of the cells. (C) and (D) the light scattering spectra along the scattering angle for the healthy RBC in (A) and the $P f$-RBC in (B), respectively.

The diluted RBC samples were sandwiched between two cover glasses, and then the diffracted beams were measured using the off-axis interferometer with the He-Ne laser $(\lambda=$ $633 \mathrm{~nm}$ ), as explained in Section 3.1. The ARLS signals from individual RBCs and $P f$-RBCs at ring stage are shown in Figs. 5(A) and 5(B), respectively. For a healthy RBC [Fig. 5(A)], the sFTLS signal shows the characteristic oscillatory peaks up to $80^{\circ}$, which is consistent with the previous result from the numerical simulation. However, FTLS measured scattering spectra only up to $71^{\circ}$ (theoretical limit) and the FTLS signal beyond $25^{\circ}$ suffers from considerably low SNR. The sFTLS signal of the $P f$-RBC exhibits the significant SNR enhancement of the measured scattering signal as well as extended measurement angle range. As can be seen in Fig. 5(B), the FTLS signal beyond $10^{\circ}$ suffers from considerably low SNR whereas the sFTLS signal measured from the same cell shows scattering signals up to $40^{\circ}$. This is because the amount of light scattering from $P f$-RBC is reduced due to the decrease in refractive index of the cell cytoplasm, which is caused by the degradation of the hemoglobin protein by malaria parasites.

\section{Conclusions}

We have presented a novel light scattering spectroscopy method, sFTLS, in order to measure ARLS signal from microscopic individual samples. Using the principle of aperture synthesis and a FTLS technique, we demonstrated that ARLS from individual objects can be quantitatively and precisely measured over an extended range of light scattering. To achieve this goal, we introduced the concept of selective aperture synthesis to solve the thick sample problem. We also measured the ARLS signals from both individual microstructures and live 
biological cells (individual RBCs and $P f$-RBCs) up to a scattering angle of $90^{\circ}$, for the first time to our knowledge. For comparison, the ARLS signals were retrieved using both the FTLS and sFTLS techniques. Using sFTLS, we measured ARLS signals over the extended range of scattering angles, whereas the maximum observable angle in the conventional FTLS was fundamentally limited by the NA of the objective lens. Furthermore, sFTLS provided significantly enhanced SNR due to the synthesis of multiple apertures. The present method will expand up potential applications of FTLS, from the characterization of individual particles at the micro- and sub-micrometer scale [34], to the investigation of subtle alterations in the light scattering signals in biological cells associated with human diseases [35-37].

\section{Acknowledgments}

This work was supported by KAIST, and the Korean Ministry of Education, Science and Technology (MEST) grant No. 2009-0087691 (BRL) and National Research Foundation (2012R1A1A1009082, 2012K1A31A1A09055128, M3C1A1-048860, 2013M3C1A3000499). YKP acknowledges support from TJ ChungAm Foundation. We thank Dr. Monica Diez (Massachusetts Institute of Technology, USA) for providing the PfRBC samples. 